\title{
Neuroimaging in psychiatry
}

Dara M Cannon

Ir J Psych Med 2007; 24(3): 86-88

The introduction of brain imaging technology revolutionized the study of psychiatric illnesses and continues to advance our understanding of the biological abnormalities that underpin psychiatric disorders. Aside from ruling out 'organic' brain pathology, neuroimaging modalities do not yet have a major role in the diagnosis of psychiatric disorders; however neuroimaging has begun to aid in the identification of disease specific intermediate phenotypes (or endophenotypes) which may be useful in aiding diagnosis and in the prediction of treatment response in psychiatric illnesses. The greatest potential of neuroimaging as an approach lies in its combined use with carefully defined psychiatric phenotypes, genotyping, neuropsychopharmacology and post-mortem studies. In this way, neuroimaging can provide a means by which preclinical data can be translated into clinically useful information for application in psychiatry. This editorial is intended to provide a brief overview of the range of imaging modalities and methods currently being applied in psychiatry and includes examples of resulting data.

Thirty years ago the advent of $x$-ray computed tomography (CT) transformed medical diagnostics.' In psychiatry, these first CT studies assessed cerebral and ventricular volume and sulcal size. The most consistent findings from these studies were that patients with schizophrenia and mood disorders demonstrated ventricular enlargement and sulcal widening, albeit to differing degrees. In addition, early MRI studies identified increasing white matter hyperintensities $(\mathrm{WMH}$; bright foci observed on T2 MRI scans thought to represent injury to white matter tracts), with age and their association with cerebrovascular disease, bipolar disorder and late-onset depression. Unlike these early imaging studies, more recent studies have begun to identify disease specific pathophysiological abnormalities.

Structural MRI studies have more commonly examined the differences in grey matter volume in psychiatric populations. Areas of the brain strongly implicated in emotion regulation and cognitive processing have been reported to have reduced grey matter volume during depression including for example, the subgenual anterior cingulate cortex ${ }^{2}$ and hippocampus. ${ }^{3}$ Altered grey matter volume has also been identified in schizophrenia in prefrontal and temporal cortical areas $^{4,5}$ and in bipolar disorder in several areas implicated in mood regulation. ${ }^{6}$ Structural MR images can also be used to

Dara M Cannon, PhD, Department of Psychiatry, 201 Comerford Building, Clinical Sciences Institute, National University of Ireland, Galway, Ireland. E-mail: dara.cannon@nuigalway.ie SUBMITED: AUGUST 1, 2007. ACCEPTED: AUGUST 27, 2007. assess cortical thickness, curvature, surface area and white matter volume. First episode schizophrenia has recently been associated with reduced thickness of the paralimbic area of the anterior cingulate cortex concomitant with an increase in the surface area. ${ }^{7}$ These newer methods of examining brain structure add significantly to the pool of knowledge that can be gained from structural MR imaging studies in psychiatry and are likely to be more widely used in upcoming studies.

In addition to structural MR-based studies, the MRI scanner can also be used to perform spectroscopy, and diffusion tensor imaging (DTI). The most commonly used form of MR spectroscopy is proton spectroscopy (1H-MRS). 1H-MRS can be used to assess levels of a number of molecules and neurotransmitters in the brain including glutamate, GABA, and markers of neuronal and glial integrity. Reductions in GABA levels in occipital and frontal cortices have been reported during depression, however, it is less clear whether this deficit exists following remission. ${ }^{8,9}$ In addition, the majority of MRS studies during depression have reported increased membrane turnover without neurodegeneration and in contrast to bipolar disorder (BD), a hypoglutamatergic state in major depressive disorder (MDD). ${ }^{10,11} \mathrm{DTI}$ is a relatively new $w^{12}$ and rapidly developing diffusion imaging method which exploits the tendency of water molecules in the axonal fibres of the myelinated or white matter tracts to diffuse preferentially in one direction, a property known as anisotropy. Changes in white matter tract integrity can thereby be detected and disruptions to the tracts are thought to represent altered connectivity between regions. However the microstructural abnormalities underlying diffusion differences are unknown. The majority of DTI studies in schizophrenic samples have detected relatively widespread alteration in brain white matter integrity. ${ }^{13,14}$ The most rapidly developing aspect of DTI currently is the computational modeling of the diffusion signal which may have contributed to inconsistencies between studies observed to date.

The combined discoveries and efforts of chemists, physicists and medical investigators have lead to the development of single photon emission computed tomography (SPECT), and positron emission tomography (PET). These revolutionary new technologies have permitted the direct visualisation of tumours, neuronal degeneration, and localisation of origin points of seizures in epileptic subjects. The first functional neuroimaging studies exploited the ability of $P E T$ with radiolabelled water (15O-H2O), and deoxyglucose (18F-FDG) to measure the rate of cerebral blood flow (CBF) and the rate of glucose metabolism, respectively. These represented the first imaging methods of assessing the neurobiological correlates of human behaviour. These functional studies have given us a myriad data, for example implicating reduced blood flow and metabolism during clinical depression in the subgenual 
anterior cingulate cortex..$^{15}$ The most common functional imaging method applied presently, however, is functional MRI ( $\mathrm{MRI}$ ), which affords greater temporal resolution of cerebral activity than PET, for example it can take an entire brain measurement at the time a volunteer is experiencing anticipation of reward and a second entire brain measurement moments later during the subjective experience of reward itself. ${ }^{16}$ One of the most novel discoveries made recently using $\mathrm{fMRI}$ was of awareness in a vegetative state. ${ }^{17} \mathrm{~A}$ young female subject in a vegetative state as a result of a car accident was asked to imagine playing tennis or moving about her home: the cortical areas activated in response to this instruction were indistinguishable from those activated by healthy volunteers.

Functional imaging studies assessing cerebral blood flow, glucose metabolism and the $\mathrm{fMRl}$ signal are global measures representing synaptic activity but in a non-specific manner. A vital step to capitalize on the evidence derived from these methods is to examine what may underpin altered neuronal activity at an anatomical and/or molecular level. The widening array of targets one can assess using molecular PET and SPECT make this possible. Molecular PET / SPECT radioligands have binding characteristics that enable estimation of an outcome parameter that is proportional to the density of the target, such as a receptor or a transporter. This molecular imaging has lead to the discoveries of altered levels of several proteins during depression including the serotonin transporter ${ }^{18,19}$ the serotonin-1A receptor ${ }^{20}$ either reduced dopamine-2 receptor levels or increased dopamine release ${ }^{21}$ and reduced cholinergic muscarinic- 2 receptors ${ }^{22}$ in several brain areas involved in emotion regulation in each case. The meaningfulness of these data are heavily dependent on certain vital sample characteristics including homogeneity of mood state, medication-free status, diagnostic subtype and the inclusion/exclusion of comorbid states. Controlling for these factors can lead to the detection of molecular differences between phenotypically similar disorders. The level of the serotonin transporter in the brainstem of depressed unmedicated individuals with MDD differs from that in depressed unmedicated individuals with BD. This could conceivably underlie the observed difference in antidepressant efficacy of serotonin reuptake inhibitors in MDD, which is approximately $75 \%$, relative to that in $\mathrm{BD}$, about $35 \% .^{23}$ Evidence supporting this has recently emerged. A better response to the serotonin reuptake inhibitor, citalopram in the STAR-D study was associated with a polymorphism in the gene that codes for the $5-\mathrm{HT} 2 \mathrm{~A}$ receptor ${ }^{24}$ and this same polymorphism was associated with having greater levels of the serotonin transporter binding in the above mentioned depressed sample (pending publication). The extension of these types of preliminary data to larger samples may help to identify endophenotypes or a set of characteristics that indicate the likelihood that a subgroup of individuals possessing such characteristics will respond preferentially to a given treatment.

In molecular PET and SPECT imaging, certain radioligands are sensitive to displacement by the endogenous neurotransmitter. This permits the measurement of neurotransmitter release, such as in the case of $\mathrm{C}-11$ labelled raclopride which can be used to assess dopamine release. Martin-Soeich et al (2006) have recently shown that a natural reward task involv- ing gambling with no pharmacological intervention, results in adequate dopamine release to measurably displace raclopride binding. ${ }^{25}$ These data demonstrate that naturalistic paradigms may be combined with imaging to further extend our understanding of the biochemical alterations associated with addiction or anhedonia. (These data will be presented at the 'Neuroscience Ireland' meeting in August 2008 in NUI Galway.) Unfortunately there is no molecular radioligand sensitive to displacement by serotonin currently available for use in humans. One can additionally measure target occupancy using PET which has proven useful in determining the level of serotonin transporter occupancy necessary to achieve clinical efficacy. ${ }^{26}$ These data are subsequently helpful in the early elimination of agents which have inappropriate levels of side effects in animals when administered at the level of occupancy necessary to achieve an antidepressant response.

To date, the discovery of therapeutic agents in psychiatry has been somewhat serendipitous such as the mood stabilizing effects of lithium and the anticonvulsants in the treatment of bipolar disorder. It is critical that novel therapeutic targets are identified and that further investigations into the prediction of treatment response be carried out. Many individuals have an unsatisfactory response to existing treatment options and/or become disheartened with multiple pharmacological trials. The majority of imaging studies aimed at identifying predictors of treatment response to date have focused on MDD and obsessive compulsive disorder (OCD). These studies have most commonly compared glucose metabolism assessed using FDG-PET in response to task performance before and after treatment response (for example see ref 27). Although these data are of limited clinical utility currently, they represent some of the first steps toward the development of neuroimaging as a clinical tool in psychiatry. The potential for clinical application of PET is illustrated further by a recently developed PET radioligand, Pittsburgh Compound- $\mathrm{B}(\mathrm{PiB})$. This radioligand binds amyloid and therefore serves as a means to monitor progression of Alzheimer's disease, and consequently treatment performance in retarding disease progression.

It is unlikely that preclinical studies alone, such as animal models and probing second messenger systems, will lead to a comprehensive understanding of the pathophysiology of psychiatric illnesses. However, these studies critically provide the evidence necessary to design translational studies. Neuroimaging provides a means by which such preclinical data can be translated into clinically applicable information. The major advantages of the neuroimaging approach are that it can be carried out in a relatively non-invasive manner, can obtain data throughout the entire brain, and that these data can be obtained during subjective experiences in human volunteers. The resolution of PET and SPECT and the number of molecular targets that can be assessed, while not ideal has improved greatly, but these do not match that of a post-mortem approach. On the other hand, the time consuming post-mortem approach can select only a small area of the brain to assess a given molecular target. Thus it appears optimal to combine the use of neuroimaging tools and post-mortem approaches to identify molecular abnormalities associated with the disease state. In this way, the molecular pathology underlying abnormalities identified using molecu- 
lar neuroimaging can be examined further. For instance, functional imaging studies have strongly implicated the amygdala in anxiety and depression and subsequent post-mortem histological studies identified reductions in glial cells with no change in neuronal cell numbers in MDD. ${ }^{28}$ Similarly, reduced grey matter volume of the hippocampus during depression detected using MRI, has been informed by post-mortem studies revealing changes in the mRNA levels of factors important to neuronal cell growth and survival. ${ }^{29}$ In combination with genetic information and functional correlates these combined approaches represent a powerful way to biologically characterise the complex array of contributors to a disease state in psychiatry.

There is currently a burgeoning degree of neuroimaging research in Ireland. Trinity College Institute of Neuroscience (TCIN, www.tcd.ie/Neuroscience/) operates a research dedicated MRI scanner for a range of functional MRI studies. In Galway, our Clinical Neuroimaging Lab at NUIG (www.nuigalway.ie/psychiatry/research/neuroimaging_lab/) is involved in structural MRI studies, MR spectroscopy, and is developing DTI based research in psychiatric populations. A research dedicated MRI scanner is planned at St. James' Hospital. Researchers in Beaumont Hospital, St. Vincent's University Hospital and the AMNCH in Tallaght are also engaged in neuroimaging studies.

The neuroimaging community in Ireland is expanding expertise in a growing number of imaging modalities. In addition to adult psychiatry these are currently being applied to disciplines including cognitive psychology, neurology, pediatric, and old-age psychiatry through a number of productive national and international collaborations. Structural MR studies of individuals experiencing their first episode of psychosis or with euthymic bipolar disorder are underway at NUI Galway. These studies additionally aim to examine the genetic contribution to morphometric abnormalities in collaboration with Prof. Michael Gill in Trinity College Dublin. Molecular PET imaging collaborations with the Drs. William Theodore and Wayne Drevets at NIH in the US, involve assessing serotonin transporter levels in subjects with temporal lobe epilepsy. Kinetic modeling of the PET data to produce images of serotonin transporter binding is preformed by the Clinical Neuroimaging Lab at NUI Galway.

The data described in this editorial are intended to convey that neuroimaging can provide a critical methodological bridge between preclinical and clinical investigations, and to identify biologic targets for the quantitative assessment of treatment response and of variables influencing illness progression. The Irish neuroimaging community are well positioned presently, to advance the understanding of the biological basis of clinical disorders including psychiatric illnesses through initiating and funding multidisciplinary studies in translational medicine.

\section{Declaration of interest: None}

*More information about our studies is available at: www.nuiga/way.ielpsychiatry/research/neuroimaging_lab/

\section{References}

1. Hounsfield, G.N., Computerized transverse axial scanning (tomography). 1. Description of system. Br J Radiol, 1973. 46(552): p. 1016-22.

2. Drevets, W.C., D. Ongur, and J.L. Price, Neuroimaging abnormalities in the subgenual prefrontal cortex: implications for the pathophysiology of familial mood disorders. Mol Psychiatry, 1998. 3(3): p. 220-6, 190-1.

3. Macmaster, F.P., et al., Amygdala and Hippocampal Volumes in Familial Early Onset Major Depressive Disorder. Biol Psychiatry, 2007

4. Okugawa, G., C. Tamagaki, and I. Agartz, Frontal and temporal volume size of grey and white matter in patients with schizophrenia : An MRI parcellation study. Eur Arch Psychiatry Clin Neurosci, 2007.

5. McDonald, C., et al., Regional volume deviations of brain structure in schizophreniz and psychotic bipolar disorder: computational morphometry study. Br J Psychiatry, 2005. 186: p. 369-77.

6. McDonald, $C$., et al., Meta-analysis of magnetic resonance imaging brain morphometry studies in bipolar disorder. Biol Psychiatry, 2004. 56(6): p. 411-7.

7. Fornito, A., et al., Surface-based morphometry of the anterior cingulate cortex in first episode schizophrenia. Hum Brain Mapp, 2007.

8. Sanacora, G., et al., Reduced cortical gamma-aminobutyric acid levels in depressed patients determined by proton magnetic resonance spectroscopy. Arch Gen Psychiatry, 1999. 56(11): p. 1043-7.

9. Bhagwagar, $Z$., et al., Reduction in occipital cortex gamma-aminobutyric acid concentrations in medication-free recovered unipolar depressed and bipolar subjects. Biol Psychiatry, 2007. 61(6): p. 806-12.

10. Yildiz-Yesiloglu, A. and D.P. Ankerst, Review of $1 \mathrm{H}$ magnetic resonance spectroscopy findings in major depressive disorder: a meta-analysis. Psychiatry Res, 2006. 147(1): p. 1-25.

11. Yildiz-Yesiloglu, A. and D.P. Ankerst, Neurochemical alterations of the brain in bipolar disorder and their implications for pathophysiology: a systematic review of the in vivo proton magnetic resonance spectroscopy findings. Prog Neuropsychopharmacol Biol Psychiatry, 2006. 30(6): p. 969-95.

12. Basser, P.J., J. Mattiello, and D. LeBihan, Estimation of the effective self-diffusion tensor from the NMR spin echo. J Magn Reson B, 1994. 103(3): p. 247-54.

13. Fujiwara, $\mathrm{H}_{\text {., }}$ et al., Anterior and posterior cingulum abnormalities and their association with psychopathology in schizophrenia: A diffusion tensor imaging study. Schizophr Res, 2007.

14. Mori, T., et al., Progressive changes of white matter integrity in schizophrenia revealed by diffusion tensor imaging. Psychiatry Res, 2007. 154(2): p. 133-45.

15. Drevets, W., et al., Subgenule prefrontal cortex abnormalities in mood disorders. Nature (Letters to Nature), 1997. 386: p. 824-827.

16. Knutson, B., et al., Dissociation of reward anticipation and outcome with eventrelated fMRI. Neuroreport, 2001. 12(17): p. 3683-7.

17. Owen, A.M., et al., Detecting awareness in the vegetative state. Science, 2006 313(5792): p. 1402

18. Cannon, D.M., et al., Serotonin transporter binding in bipolar disorder assessed using [1 1C]DASB and positron emission tomography. Biol Psychiatry, 2006. 60(3): p. 207-17.

19. Meyer, J.H., et al., Brain serotonin transporter binding potential measured with carbon 11-labeled DASB positron emission tomography: effects of major depressive episodes and severity of dysfunctional attitudes. Arch Gen Psychiatry, 2004. 61(12): p. $1271-9$.

20. Drevets, W.C., et al., Serotonin type-1A receptor imaging in depression. Nucl Med Biol, 2000. 27(5): p. 499-507.

21. Montgomery, A.J., et al., Extrastriatal D2 and striatal D2 receptors in depressive illness: pilot PET studies using [11C]FLB 457 and [11C]raclopride. J Affect Disord, 2007. $101(1-3):$. 113-22.

22. Cannon, D.M., et al., Reduced muscarinic type 2 receptor binding in subjects with bipolar disorder. Arch Gen Psychiatry, 2006. 63(7): p. 741-7.

23. Cannon, D.M., et al., Elevated Serotonin Transporter Binding in Major Depressive Disorder Assessed Using Positron Emission Tomography and [(11)C]DASB; Comparison with Bipolar Disorder. Biol Psychiatry, 2007.

24. McMahon, F.J., et al., Variation in the gene encoding the serotonin 2A receptor is associated with outcome of antidepressant treatment. Am J Hum Genet, 2006. 78(5): p. 804-14.

25. Martin-Soelch, C., et al., Lateralized dopaminergic response to reward in the human ventral striatum. Society for Neuroscience Annual Meeting Abstract Book, 2007.

26. Meyer, J.H., et al., Serotonin transporter occupancy of five selective serotonin reuptake inhibitors at different doses: an [11C]DASB positron emission tomography study. Am J Psychiatry, 2004. 161(5): p. 826-35.

27. Saxena, S., et al., Differential cerebral metabolic changes with paroxetine treatment of obsessive-compulsive disorder vs major depression. Arch Gen Psychiatry, 2002. 59(3): p. 250-61.

28. Hamidi, M., W.C. Drevets, and J.L. Price, Glial reduction in amygdala in major depressive disorder is due to oligodendrocytes. Biol Psychiatry, 2004. 55(6): p. 563-9. 29. Gaughran, F, et al., Hippocampal FGF-2 and FGFR1 mRNA expression in major depression, schizophrenia and bipolar disorder. Brain Res Bull, 2006. 70(3): p. $221-7$ 


\section{Unlock your potential...}
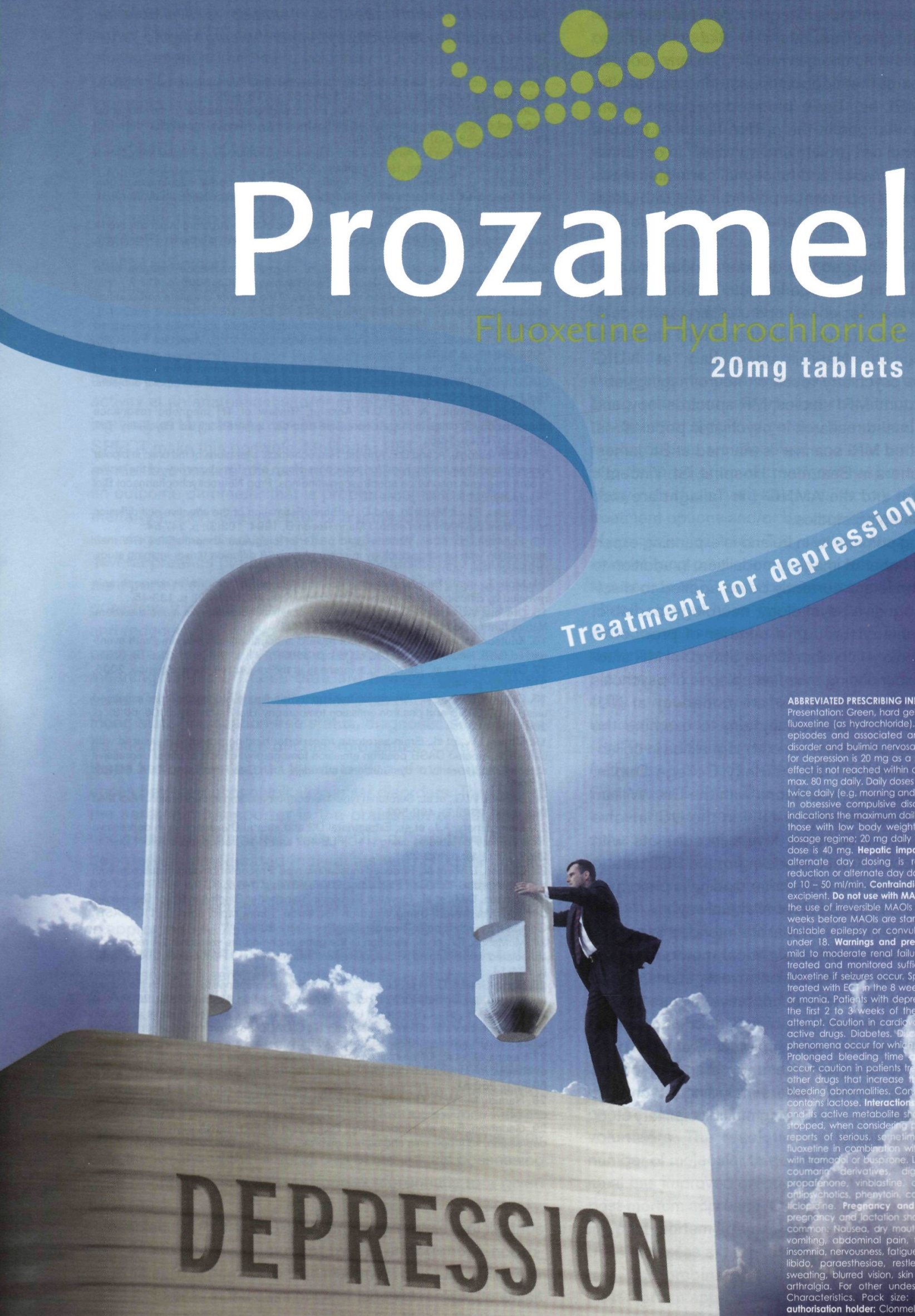

$20 \mathrm{mg}$ tablets

debrese

ABBREVIATED PRESCRIBING INFORMATION PTozamel $20 \mathrm{mg}$ Capsules fivoxe tine (as hydrochloride). Indications: Treatment of major depressive episodes and associated anxiety. Treatment of obsessive compulsive for deoression is $20 \mathrm{mg}$ as a single dose in the morning. If the expected effect is not reached within a few weeks, the dose can be increased to thity In obsessive compulsive disorder the dose is $20-60 \mathrm{mg}$ those with low body weight should be treated with an dosage regime; $20 \mathrm{mg}$ daily is usually well tolerated. The me alternaie day dosing is recommended. Renal impairme reduction or alfernate day dosing is recommended for pati of $-50 \mathrm{~m} / \mathrm{min}$. Contraindications: Hypersensinily to hud

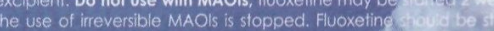
weeks beiore MAOls are starled. Severe renal fo, 1 (c) $R<10 \mathrm{~m} / \mathrm{min}$ under 18 . Warnings and precautions: Severe he Polients with stah?

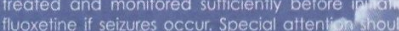
treated with EC $h$ the 8 weeks befor fuxetine treorinent th pomania

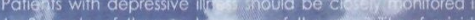

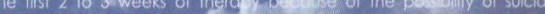
allempt. Caution in cardid asougo obsese, patients on lithium or CNS

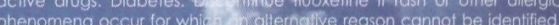
Prolonged bleeding time caution in patients try 16 d concomitantiy with anticoagulants or other drugs that increase f risk of bleeding potients with previous

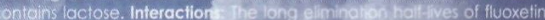

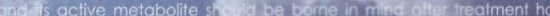
topped. when considerita poss ble dug interaclion. there have been

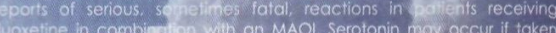
with tramarf or buspirone. Linyptophan, diazepam, cimelidine, lithium,

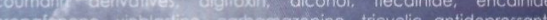

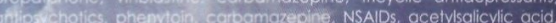
felop, ine. Pregnancy and laclation: The use of fluoxetine during pregenancy and flactation should be avoided. Undesirable effects: Mo

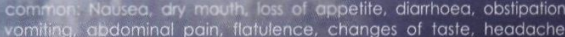
arvousness, fatique, tremor. dizziness, impotence, reduction of esiae, restlessness, nightmares, weakness, excessive

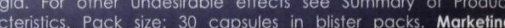
(a) authorisation holder: Clonmel Healthcare Ltd, Waterford Road, Clonme, $126 / 110 / 1$. Full prescribing information is available on request. Date las evised: February 2007 